\title{
REVIEW
}

\section{Intra-Abdominal and Abdominal Wall Desmoid Fibromatosis}

\author{
J. Harrison Howard (D) Raphael E. Pollock
}

To view enhanced content go to www.oncologytherapy-open.com Received: November 23, 2015 / Published online: February 3, 2016

(C) The Author(s) 2016. This article is published with open access at Springerlink.com

\section{ABSTRACT}

Desmoid fibromatosis is a rare but locally aggressive tumor comprised of myofibroblasts. Desmoids do not have the ability to metastasize but can cause significant morbidity and mortality by local invasion. These tumors may occur throughout the body, but are commonly found on the abdominal wall and within the intestinal mesentery. Desmoids in these areas may cause unique clinical problems for physicians and patients. Mutations in either the $\beta$-catenin or the APC genes are usually the cause for the development of these tumors with the former comprising the sporadic development of tumors and the latter being associated with familial adenomatous polyposis syndrome. Surgical resection with histologically

Electronic supplementary material The online version of this article (doi:10.1007/s40487-016-0017-z) contains supplementary material, which is available to authorized users.

J. H. Howard $(\bowtie) \cdot$ R. E. Pollock

Division of Surgical Oncology, The Ohio State

University Wexner Medical Center, Arthur G. James

Cancer Hospital and Richard J. Solove Research

Institute, M256 Starling Loving Hall, 320 W. 10th

Ave, Columbus, OH 43210, USA

e-mail: john.howard@osumc.edu negative margins has been the cornerstone of therapy for this disease, but this paradigm has begun to shift. It is now common to accept a microscopically positive margin after resection as recurrence rates may not be significantly affected. An even more radical evolution in management has been the recent movement towards "watchful waiting" when new desmoids are diagnosed. As the natural history of desmoids has become better understood, it is evident that some tumors will not grow and may even spontaneously regress sparing patients the morbidity of more aggressive therapy. Other modalities of treatment for desmoids include radiation and systemic therapy which both can be used adjuvantly or as definitive therapy and have shown durable response rates as single therapy regimens. The decision to use radiation and/or systemic therapies is often based on tumor biology, tumor location, surgical morbidity, and patient preference. Systemic therapy options have increased to include hormonal therapies, non-steroidal anti-inflammatory drugs and chemotherapy, as well as targeted therapies. Unfortunately, the rarity of this disease has resulted in a scarcity of randomized trials to 
evaluate any of these therapies emphasizing the need for this disease to be treated at high volume multidisciplinary institutions.

Keywords: Abdominal desmoid tumor; Abdominal wall desmoid tumor; Desmoid fibromatosis; Desmoid tumor

\section{INTRODUCTION}

Desmoid tumors present a difficult problem for both physician and patient. While many options for treatment are available, the natural history of this disease can be unpredictable resulting in a complex, multidisciplinary decision-making process with treatment algorithms that include combinations of radical surgery, systemic therapy and radiation, but may be as minimal as close observation. As a disease that has been referred to as a "benign malignancy" it may be challenging for patients to understand why such aggressive measures are needed for a disease that does not metastasize. Conversely, patients may be anxious about observing a tumor that has potential to grow and become locally invasive. These tumors can occur nearly anywhere on the body, but this discussion will focus on the biology and treatment of abdominal wall desmoids and intra-abdominal desmoid tumors.

This article is based on previously conducted studies and does not involve any new studies of human or animal subjects performed by any of the authors.

\section{NATURAL HISTORY}

Desmoid tumors are rare with an incidence of $0.03 \%$ of all neoplasms and 3\% of soft tissue tumors [1]. The majority of desmoid tumors (also known as aggressive fibromatosis) occur sporadically, but can be associated with familial adenomatous polyposis syndrome (FAP). FAP-associated desmoid tumors occur in $2-15 \%$ of all desmoid patients, and are typically located in the small bowel mesentery [2-5]. Desmoids are seen more commonly in women than men with a 2:1 predilection for females $[4,6,7]$. Young adult populations are most commonly affected and this disease is frequently seen in the 25-35 year old demographic $[4,6]$.

Histologically, desmoid tumors are a benign proliferation of myofibroblasts without a capsule that locally infiltrates surrounding tissues and may be multifocal. These characteristics result in a tumor that is difficult to control locally, but does not have the ability to metastasize [8-10]. Desmoids not only often occur on the abdominal wall or the mesentery of the small intestine, but also affect the shoulder girdle, chest wall and extremities. These tumors are often thought of as a chronic disease and require close observation regardless of treatment strategy as they have potential for mortality due to their locally aggressive behavior and ability to invade adjacent critical organs and structures [3].

\section{RISK FACTORS AND GENETIC PREDISPOSITION}

The majority of cases of abdominal wall desmoid tumor are sporadic in nature, but there are several associated factors. Development of desmoids is most commonly associated with genetic predisposition, pregnancy, hormonal exposure, and physical factors such as trauma and/or surgery [6, 7]. A classic presentation of desmoid includes a post-partum woman with an abdominal wall mass. Given the identification of estrogen receptors on desmoid tumors, a predilection 
for women and the use of anti-estrogen therapies to manage these tumors, there does seem to be validity in the involvement of estrogen in disease progression $[3,11]$. While there is no clear relationship between pregnancy and abdominal wall desmoid tumors, a recent study of pregnancy-related desmoid fibromatosis showed that these tumors have an indolent course, do not increase risk of obstetric complications and should not be a contraindication for subsequent pregnancies [12]. This study did note that in women diagnosed with desmoid tumor prior to becoming pregnant there was a $42 \%$ chance of either disease recurrence or progression during pregnancy, suggesting a different biology than disease that developed during or after pregnancy. For women who developed desmoid tumor associated with pregnancy, 52\% were managed successfully without surgical resection, and a local relapse of $13 \%$ was observed for those who did have surgical resection. Ultimately, the study concluded that desmoid fibromatosis that develops or progresses during pregnancy is safely managed with minimal risk to both mother and child.

Several other patient-associated risk factors have been studied with regard to prognosis. Specifically patient gender, tumor size, tumor site, and presentation (primary vs recurrent) have been correlated with clinical outcomes. In a multi-institutional retrospective review, Peng et al. found that younger age and extra-abdominal tumor location were associated with poorer recurrence-free survival [13]. Several studies have confirmed that young age, large tumor size, and extra-abdominal location are predictors of poor outcomes. Patient sex and primary vs recurrent disease were not prognostic for recurrence [14, 15]. Conversely, other studies have correlated recurrent disease with having worse 5- and 10-year event-free survival when compared to new primary tumors [16, 17]. While not reaching statistical significance, Lev et al. also found that age of $<30$ years and extremity tumor site were associated with higher recurrence rates [18]. As larger series becomes available, it seems that younger age, large tumor size, and anatomic location may play a true role in defining recurrence risk for desmoid tumors. Based on these data, abdominal wall desmoids are likely to have a more favorable outcome. Beyond these classic clinical prognostic factors, a better understanding of the genetic fingerprint of desmoids may be more reliable in stratifying disease aggressiveness. Studies have begun to identify gene expression profiles that may better predict tumor biology [19].

Alterations in the Wnt signaling pathway have been identified as the likely driving mechanism of tumorigenesis in the development of desmoid fibromatosis [20, 21]. In general, deregulation of the Wnt pathway results in aberrant proliferation, migration, and differentiation of cells into human cancers [20]. Two specific genetic mutations that alter this pathway and are associated with the development of desmoid tumors have been identified: the CTNNB1 gene, coding for the $\beta$-catenin protein, and the $A P C$ gene, coding for the adenomatous polyposis coli (APC) protein. Both $\beta$-catenin and APC are part of the Wnt pathway suggesting that two separate mutations affecting the same endpoint are involved with the development of desmoid fibromatosis [3]. These mutations result in the development of intranuclear accumulation of $\beta$-catenin which subsequently stimulates DNA transcription and cell proliferation [21]. Eighty-five percent of sporadic desmoid tumors have been identified to have an 
activating mutation in the CTNNB1 gene coding for $\beta$-catenin. The germline mutation of the APC gene leads to the development of desmoid tumors for FAP patients [21, 22]. A recent genetic analysis study by Crago et al. looking at "wild type" desmoids without the known CTNNB1 or APC mutations found that with deep sequencing 95\% of desmoids may have mutations that affect the $\mathrm{Wnt} / \beta$-catenin pathway, suggesting a near universal relationship between desmoid tumors and Wnt signaling [23].

For sporadic disease, there is a high rate of mutation in the gene encoding for $\beta$-catenin on chromosome 3, CTNNB1. CTNNB1 mutations have been found in $71-91 \%$ of sporadic desmoid tumors with the highest rate of mutation found in intra-abdominal tumors [21, 24-28]. The most commonly documented mutations found on the gene are $\mathrm{T} 41 \mathrm{~A}$ and S45F, with the latter being nearly exclusive to extra-abdominal desmoids. Several studies have shown a significantly higher chance of disease recurrence at 5 years despite complete resection of disease for patients harboring an $\mathrm{S} 45 \mathrm{~F}$ mutation [21, 26, 28]. Domont et al. found higher recurrence rates for tumors with CTNNB1 mutations, but could not correlate this finding with any specific point mutations. The growing amount of data suggests that specific mutations within this gene do play a role in disease recurrence and may influence clinical care in the future based on the tumor's genetic fingerprint.

For patients with wild type $\beta$-catenin, the mutated APC gene is suspected to be the source of development of desmoid tumors. While patients with FAP represent the minority of desmoid cases, patients with this syndrome have nearly an 850-fold increased chance of development of desmoids compared to the general population. Approximately $10-15 \%$ of patients with FAP develop desmoids and it has become the primary cause of death in patients with FAP that have previously had a prophylactic colectomy [22, 29]. As a tumor suppressor gene, when $A P C$ is mutated it is unable to properly regulate $\beta$-catenin levels through the Wnt pathway leading to proliferation and accumulation of $\beta$-catenin in cell cytoplasm and nucleus. Interestingly, despite a similar molecular endpoint between APC and $\beta$-catenin mutations, the phenotype of APC-mutated tumors is different as the majority of tumors in patients with FAP are intra-abdominal and involving the small bowel mesentery [2].

\section{TREATMENT}

\section{Surgery}

Margin-negative resection has historically been the gold standard for treatment of abdominal wall and intra-abdominal desmoid tumors. However, as a better understanding of the natural history of desmoids developed, strategies for surgical resection have evolved. The initial surgical approach has transitioned from margin-negative resection to acceptance of microscopically-positive resection to observation with surgery used more selectively.

\section{Margin Status}

When surgical resection is employed for desmoid tumors, many clinicians encourage complete microscopic resection $[5,13,17,30]$. However, unlike soft tissue sarcoma where positive margin status is a clear indicator of disease recurrence, there are conflicting data on the significance of complete microscopic resection of desmoid tumors (Table 1). Large retrospective studies have shown higher 


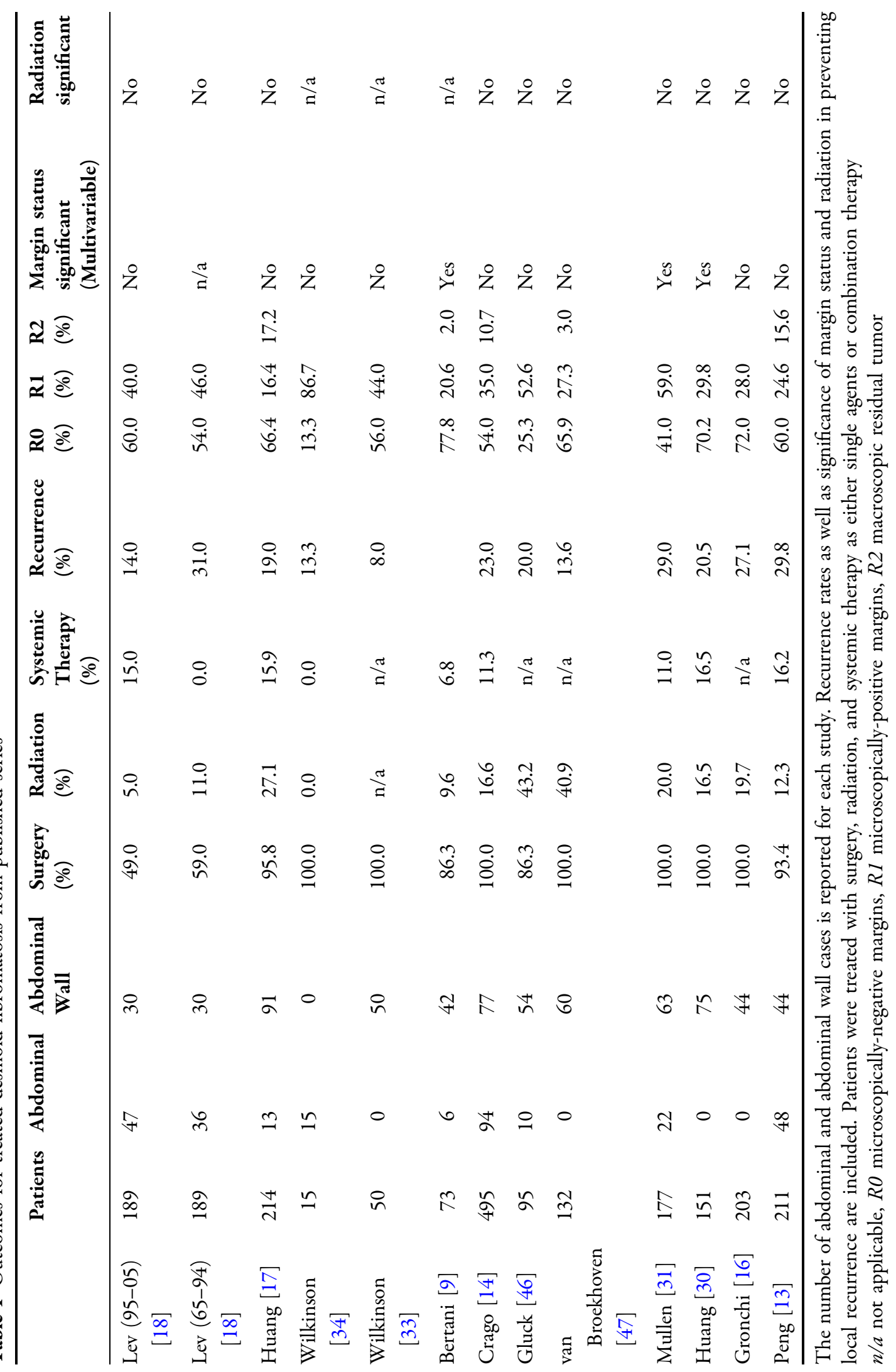


recurrence rates for patients with microscopically-positive margins (R1) when compared to microscopically negative (R0) margins [13, 17, 18, 30, 31]. However, some studies have only shown margin status as a predictor of recurrence with univariate analysis but not on multivariate analysis [13, 31]. Other studies show that microscopic margin status does not affect recurrence-free survival and has no prognostic significance when comparing R0 and $\mathrm{R} 1$ resections in desmoid tumors $[14,15$, $17,31]$. Of particular interest in relation to this topic is a large study from the MD Anderson Cancer Center which compared desmoid outcomes from two distinct time frames at the institution: the first is 1965-1994, and the second a more modern cohort from 1995 to 2005. Interestingly, margin status is a significant predictor of disease recurrence in the older cohort but that significance is lost in the modern group despite similar rates of margin-positive resections between the two groups (46\% vs $47 \%$ ). This adds confusion to the true significance of margin-negative resection for desmoid tumors [18]. Ultimately margin status remains a controversial topic in the management of desmoid tumors and it is often agreed that R0 resections are ideal but that with similar recurrence rates for R0 and R1 resections as well as the emergence of adjuvant therapies additional surgery may be avoided based solely on a microscopically-positive margin. This is particularly true in patients where re-resection may result in loss of body function or high operative morbidity [5]. These decisions should be made within the confines of a multidisciplinary tumor board.

Abdominal wall and intra-abdominal desmoid tumors present unique surgical challenges due to their anatomic location. Abdominal wall desmoids may require abdominal wall resection and reconstruction while intra-abdominal and mesenteric tumors may necessitate removal of a significant amount of bowel (Fig. 1). Abdominal wall desmoids have favorable outcomes when resected and it has been suggested that these lesions have a better prognosis than those involving extremity based on low recurrence rates [32]. In a study looking at 50 patients with abdominal wall desmoid fibromatosis treated with surgery, $92 \%$ of patients did not experience recurrence after a median follow up of 6 years. In this cohort $56 \%$ of patients had an R1 resection but only tumor size $(>7 \mathrm{~cm})$ was associated with recurrence. Prosthetic mesh was used in $94 \%$ of patients to repair the surgical defect. In addition to recurrence rates, complication rates were also low with no mortality and only one postoperative complication of cellulitis [33]. Peng et al. also report good outcomes for patients with abdominal wall or intra-abdominal desmoids tumors and margin negative resection (R0). Median recurrence-free survival was not reached after follow up of 25.7 months [13]. While intra-abdominal desmoids only represented $5.8 \%$ of their population, these patients also had low complication rates as compared to patients with resected extra-abdominal desmoids. Wilkinson et al. have also confirmed good prognosis for surgically-resected sporadic intra-abdominal desmoids. Of 15 patients with sporadic (non FAP associated) desmoids that were grossly resected, 13 patients did not recur with a median disease-free interval of 45 months. R0 resections were only accomplished in 2 of these 15 patients with the remaining 13 having R1 resections [34]. In a study looking at the use of surgery for mesenteric desmoids in patients with FAP, details from 16 patients were available. Small bowel resections were necessary in $87.5 \%$ of these cases with an average of $45.6 \mathrm{~cm}$ of bowel removed [35]. When treating mesenteric desmoid tumor, it is possible that a significant amount of bowel may be resected resulting in 

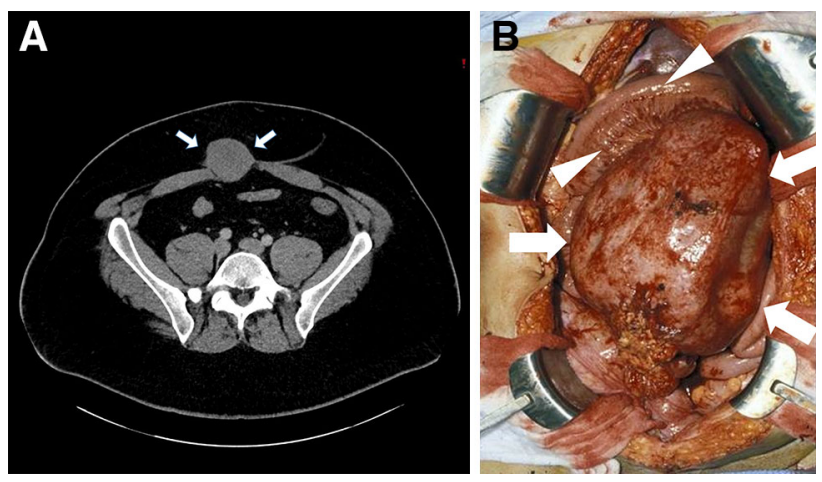

Fig. 1 Abdominal wall and intra-abdominal desmoid tumors. a Desmoid tumor of the rectus abdominis muscle (arrows) requiring full thickness resection of the abdominal wall to obtain grossly negative margins. Often this will necessitate abdominal wall reconstruction with prosthetic

intestinal failure, chronic total parenteral nutrition (TPN), and perhaps the need for small bowel transplantation. Although numbers are small (12 patients), several studies have shown encouraging results for either intestinal or modified multivisceral transplantation for patients developing short gut syndrome after resection of mesenteric desmoid tumors [36-38]. Two patients have died, but at time of publication all other patients had intestinal rehabilitation and were functioning without TPN. Long-term follow up is available for 8 patients with only 2 patients developing desmoid recurrence. Tumors recurred either in the abdominal wall, chest wall or incision without any patients developing intra-abdominal recurrence $[36,38]$. While the risks of immunosuppression and graft rejection exist for patients treated with transplant, this may represent a reasonable long-term solution for mesenteric desmoids necessitating life-altering bowel resection.

\section{Watchful Waiting}

The natural biology of abdominal wall and intra-abdominal desmoids can be unpredictable. More indolent tumors may not material. b Intra-abdominal desmoid tumors (arrows) frequently require small bowel resection due to the involvement of the mesentery, adjacent blood vessels, and bowel (arrowheads)

recur despite microscopically positive margins and more aggressive tumors may recur despite negative margins. While biologic markers are evolving, the true nature of a desmoid cannot be reliably predicted. In most large databases, there are patients who receive no treatment for their tumors and have shown no progression or sometimes even spontaneous regression. These observations have propelled a new strategy for desmoid management which is gaining traction known as "wait and see" or watchful waiting. When a new patient presents with a desmoid tumor the natural history of their disease has likely not been declared. By employing a period of close observation, proponents believe that it is possible to identify more indolent tumors, avoiding morbid procedures for tumors that may not progress or may even spontaneously regress. Conversely, by having frequent physical exams and staging scans, tumors that show more aggressive behavior can be treated with a multidisciplinary approach before the therapeutic window is missed $[5,10]$.

While evaluating the best initial therapy for primary desmoid, Bonvalot et al. identified a group of patients that were not treated 
surgically, but had a similar outcome as patients treated with R0 resection. Patients treated with observation did as well as patients with completely resected disease and better than patients with incomplete resection [39]. In an effort to understand how observation alone may affect additional therapies and outcomes for desmoid tumor, a multi-institutional study evaluated patients not treated with surgery or radiation and were stratified into an observation group or a medical therapy group. Eighty-three patients were treated with observation only and their progression was compared to patients treated systemically. The observation arm had a 49.9\% 5-year progression-free survival (PFS) which was not different from the $58.6 \%$ PFS in the systemically treated group [40]. While the authors could not identify any prognostic factors that would predict tumor progression, they were able to avoid the morbidity of surgery and/or radiation. Of patients that did progress, $89 \%$ did so in the first 2 years. Based on these findings the authors have proposed a very close regimen of disease surveillance for 24 months. The authors clearly recognize the heterogeneity of the disease and realize that proper patient selection for this strategy is critical. In an effort to better risk stratify sporadic desmoids and identify patients with more indolent tumors, Salas et al. developed a clinical risks scale based on the primary tumor characteristics. This scale has revealed prognostic subgroups that may benefit from different treatment strategies including watchful observation [15]. Several notable European groups have made proposals to standardize desmoid tumor management such that a watchful waiting strategy is the first step for all new tumors $[5,10]$. Patients will be followed closely by a multidisciplinary team with escalation of care only if the tumor is declared to be an aggressive phenotype, which spares patients with indolent tumors the morbidity of aggressive therapy. This is of particular interest for intra-abdominal desmoids where resection may lead to the removal of a significant amount of bowel. Clearly this change in management represents an opportunity for prospective trials to define the role of observation vs immediate intervention.

\section{Radiation}

Radiation has been used as both adjuvant and definitive therapy for desmoid tumors. Most data evaluating the use of radiation are from surgical studies with a clear selection bias towards offering therapy to patients that have either recurred or have had a positive margin resection. Conversely, patients that have radiation only, usually have tumors that are unresectable or would require unacceptable surgical deformity or morbidity. While radiation has a role in treating desmoid tumors, the highly selected patients treated with radiation and the lack of randomized trials make the exact role for radiation in desmoids elusive.

A large meta-analysis of patients from 22 studies treated with surgery, surgery plus radiation, or radiation alone suggested that all patients with desmoid tumors should be treated with radiation. Patients treated with radiation or radiation plus surgery had superior local control rates (78\% and $75 \%$, respectively) than patients treated with surgery alone (61\%) [41]. While supporting the role for radiation as a viable treatment of desmoid tumors, the radiation doses in this study were highly variable and declaring that all disease should be treated with this modality may be overly aggressive and does not take into consideration the morbidity associated with radiation. Complications related to radiation for desmoid 
fibromatosis are 17-23\% [41-43]. Before radiation is given for abdominal wall and intra-abdominal desmoids the risk of radiation-induced enteritis as well as the risk of radiation-induced secondary malignancies should be considered. Radiation-induced malignancies are particularly relevant in a disease that often affects a younger cohort of patients [43]. Definitive radiation may be effective for local control in patients that would otherwise need radical and disfiguring surgery. Radiation as the primary mode of therapy for unresectable disease may offer a $70-80 \%$ chance of local control [42, 44, 45]. Of particular interest is a European Organization for Research and Treatment of Cancer (EORTC) phase II study that standardized treatment of 44 patients with unresectable disease to $56 \mathrm{~Gy}$ received in 28 fractions. At 3 years, local control was $81.5 \%$ with $13.6 \%$ of patients having a complete response. They additionally report that in some patients radiation response will continue beyond 3 years, promoting the durability of this therapy for unresectable disease [44].

Studies comparing local control in surgically-resected patients based on receipt of adjuvant radiation have not been as clear. When Huang et al. reviewed their data on surgically-resected desmoids they found no overall improvement in local recurrence based on adjuvant radiation. On subset analysis, they did find a significant improvement in recurrence for patients with R1 resection who received radiation compared to those who did not. Five-year relapse free survival rates for R1 resection followed by radiation were $75 \%$ compared to $56.4 \%$ in patients with R1 resection alone [17]. While not statistically significant, Lev et al. also found a trend toward improved local control with adjuvant radiotherapy [18]. Despite some supporting evidence for the use of adjuvant radiation, many other retrospective studies have found that the use of adjuvant radiation did not impact local recurrence rates (Table 1) [14, 30, $31,46,47]$. While there seems to be a real benefit of radiation for some patients, the true utility of radiation as an adjuvant therapy in desmoid fibromatosis has not been evaluated in a prospective, randomized trial. Most studies are small, retrospective and do not have standardization of patient selection. Radiation is not frequently used for intra-abdominal disease and most studies are focused on its use for disease in the extremities and soft tissues. This is likely related to a high risk of radiation-induced enteritis and the lack of a distinct target for grossly resected disease. Due to the lack of clearly defined indications for radiation it is critical to make these decisions in large volume multidisciplinary centers to properly select patients and avoid unnecessary exposure and morbidity from this treatment.

\section{Systemic Therapy}

Studies evaluating systemic therapy for desmoid fibromatosis frequently involve patients with mesenteric desmoid tumors due to the difficulty treating this situation with other therapies. In addition to high recurrence rates, there are significant side effects and morbidity that may arise from resection of mesenteric desmoids due to involvement of major blood vessels and potential need for significant bowel resection. Many systemic therapy treatment lines have been used for desmoids including hormonal therapy, non-steroidal anti-inflammatory drugs (NSAIDs), chemotherapy, and targeted therapy with varying degrees of success but no standard of care. An example of the lack of standardized systemic therapy for desmoids is shown by 
recent retrospective studies showing up to 157 different combinations of therapy used to treat these tumors [29, 48]. Unfortunately, most studies looking at systemic therapy for treating desmoid tumors are either retrospective or single-armed studies. Additionally, they are treating patients that have failed surgical therapy or have unresectable disease, introducing selection bias and making comparisons to other therapies difficult; however these studies still provide insight into treating the disease when other options are not feasible.

\section{Hormonal and Nonsteroidal}

\section{Anti-Inflammatory Drugs (NSAIDs)}

When effectiveness, side effects, and efficacy are weighed, either combination or single agent hormonal and NSAID agents are frequently used as a first-line systemic therapy or for unresectable, recurrent, or progressing desmoid tumors $[4,10,48-50]$. Typically either tamoxifen (60-120 mg/day) or raloxifen (120-240 mg/day) is used as an anti-estrogen agent. The NSAID most commonly used is sulindac (150-800 mg/day). Hormone-based therapy are effective in $40-51 \%$ of patients $[29,51]$. Hansmann et al. observed a $77 \%$ response rate in patients with FAP-associated mesenteric desmoids treated with first line anti-estrogen/NSAID therapy. This compares favorably to patients that have recurred after surgical resection and then treated with a similar regimen; response rates in recurrent disease were only 50\% suggesting that anti-estrogen/NSAID therapy for patients with FAP-associated mesenteric desmoids may be a reasonable therapy before proceeding with surgery [49]. A recent update from the same center reports 134 patients treated with the same regimen as either adjuvant or definitive therapy. Patients with sporadic and
FAP-associated mesenteric desmoid fibromatosis were included and equally represented. A response rate of over $85 \%$ was achieved (stable disease as well as responders) using anti-estrogen/NSAID therapy. Additionally they were able to taper therapy and had only one long-term recurrence in patients that had previously been resected [52]. Such a large cohort of patients with high response rates and durability is encouraging for non-surgical management of these tumors. This combination can be effective, but an objective response may take several months to stabilize disease and decrease associated symptoms [10, $48,52]$. These agents are often used as they are relatively inexpensive and have a low-risk side effect profile compared to other systemic therapies. Despite their relatively safe reputation it should be cautioned that anti-estrogens have a slightly increased risk of thromboembolic events and tamoxifen can cause ovarian cysts in pre-menopausal women.

\section{Chemotherapy}

Several studies have found chemotherapy to be the best systemic therapy for treating desmoid tumors with response rates as high as 79\% [29, $48,53]$. There are a lack of randomized or controlled trials as most studies treat a small number of patients with heterogeneous treatment regimens. Drug combinations using anthracyclines appear to be the most effective treatment, but other combinations may additionally include methotrexate, vinblastine and cisplatin.

The anthracycline-based regimen has resulted in long progression-free survival and even complete responses. This has influenced some groups to support chemotherapy as first-line treatment for unresectable disease-particularly of the mesentery [29, 48, 53-55]. When patients show aggressive disease and fail hormonal 
therapies, chemotherapy is often the second line of therapy as it may show significant responses in previously-treated patients [10, 28, 43]. A study from the French Sarcoma Group compared anthracycline-based therapy to other regimens and were able to show a statistically significant difference in response: $54 \%$ vs $12 \%(p=0.0011)$ favoring anthracyclines. While only 13 patients were treated, all patients that received anthracycline-based therapy had either stable disease $(46 \%)$ or a partial response $(54 \%)$ [56]. Other studies have confirmed a high response rate and durable response for this regimen with progression-free survival of 74 months [53]. As might be expected, toxicity is higher with this regimen resulting in grade 3-4 hematological toxicities in approximately $31-43 \%$ of patients $[53,56]$.

Additionally, "low-dose" chemotherapy has also been described for systemic treatment of desmoids which typically includes methotrexate and vinblastine. This has been shown to be well tolerated and consistently results in stable or responding disease in $67-100 \%$ of patients treated [48, 56, 57]. Importantly, this regimen has also been associated with a prolonged response and 5 -year progression-free survival as high as $67 \%$ [57]. Unfortunately this regimen has been associated with high toxicity rates resulting in patient intolerance and a 50\% attrition rate. Neurotoxicity is a common side effect of vinblastine that often results in patients not being able to tolerate this regimen. Another vinca alkaloid, vinorelbine, has been described as an effective agent against desmoids with less long-term toxicity than vinblastine and is also given with methotrexate $[58,59]$. Weiss et al. describe a $60 \%$ response rate and improvement of symptoms of $80 \%$ of patients treated with vinorelbine in a small series of patients with previously low rates of neurotoxicity [59]. This may likely be another useful regimen particularly in patients that do not tolerate vinblastine.

\section{Targeted and Evolving Therapeutics}

Other evolving options include targeted therapies using tyrosine kinase inhibitors (TKIs) and anti-angiogenic drugs. The TKI that have been used include imatinib and sunitinib. A phase II trial using imatinib had modest results with 1-year progression-free survival of $66 \%$ and an objective response rate of only $6 \%$ [60]. Another phase II study using higher doses of imatinib (800 mg/day) revealed a $15.7 \%$ partial response (PR) rate $(\geq 50 \%$ tumor shrinkage) in a heavily pre-treated group of patients. Interestingly, all patients that experienced a PR had intra-abdominal disease and duration of response was greater than 1.5 years for all patients [61]. Finally, Penel et al. treated 40 patients with imatinib (400 mg/day) in a phase II trial and experienced $67 \%$ progression-free survival. Only $45.5 \%$ of these patients had abdominal wall or mesenteric disease, but two of these patients had a partial and durable response [62]. Toxicity for all three of these trials was acceptable with very few grade 4 toxicities that were treated effectively with dose reduction. In addition to these phase II trials, other small retrospective reviews have revealed stable or partial response in $36-80 \%$ of patients with a median progression-free survival of nearly 27 months by Response Evaluation Criteria In Solid Tumors (RECIST) criteria [43].

Given the potential activity of imatinib in the treatment of desmoid fibromatosis, another TKI, sunitinib, has been evaluated for efficacy in advanced disease. Sunitinib also blocks vascular endothelial growth factor receptors adding an anti-angiogenic effect. A phase II study treating mostly intra-abdominal desmoids $(63.2 \%$ of 
participants) with sunitinib showed an overall response rate of $26.3 \%$ and a 2-year progression-free survival rate of $74.7 \%$. Three of the 12 patients with mesenteric disease developed serious adverse events with the first dose of treatment presenting as tumor bleeding, bowel perforation and entero-tumoral fistula formation. The authors postulate that all of these events could be explained by the drugs anti-angiogenic affect with resultant tumor necrosis [63].

Additional TKI and anti-angiogenic drugs, sorafenib and pazopanib, have also been shown to have efficacy in treating desmoid tumors [64, 65]. In a small, retrospective review of 26 patients treated with sorafenib, $70 \%$ of patient reported improved symptoms, and at 6 months $95 \%$ of patients had either a partial response or stable disease. In the 13 patients with abdominal disease evaluated radiographically, nearly $73 \%$ of patients had radiographic response by RECIST criteria [64]. Following the response seen from sorafenib the anti-angiogenic drug pazopanib has also been shown to be effective in treating desmoid tumors. Two case reports show that patients treated with pazopanib had improved symptoms, tumor shrinkage and decreased tumor cellularity similar to results seen with sorafenib [65]. Conclusions regarding the efficacy of these drugs must be interpreted with caution until larger prospective clinical trials are performed to validate initial findings.

As data emerge regarding the pathophysiology of desmoid tumors, new pathways to inhibit tumor growth are being discovered. The NOTCH pathway has recently been recognized as a potential therapeutic target for desmoids. This pathway drives several cancer-related processes in solid tumors and can be blocked by $\gamma$-secretase inhibition. When desmoid tumor cell lines are treated with $\gamma$-secretase cell growth, migration and invasion are inhibited [66]. In an open label phase I dose escalation trial of a $\gamma$-secretase inhibitor, five of seven patients with desmoid tumors that were treated showed objective and durable response [67]. This promising data has spawned a phase II trial evaluating a $\gamma$-secretase inhibitor in adults with desmoid tumors [68]. Recently another target, hyaluronan (HA), a glycosaminoglycan in the stromal microenvironment involved with normal wound healing, has been identified and associated with desmoid tumorigenesis [69]. This study identified overexpression of HA levels in desmoid tumor surgical specimens as well as immortalized cell lines. When HA synthesis was inhibited, they found decreased tumor proliferation rates and decreased HA levels suggesting a novel therapeutic target in treating desmoid fibromatosis. While new targets in this difficult disease are exciting, more translational studies will be required.

\section{CONCLUSIONS}

Due to the rarity and heterogeneity of this disease, it cannot be emphasized enough that desmoid fibromatosis should be managed within the context of a high-volume, multidisciplinary tumor board. Treatment recommendations regarding surgery, radiation, and systemic therapy are all evolving. This increases the complexity of the decision making for this disease and emphasizes the necessity of having surgical oncologists, radiation oncologists and medical oncologists involved in developing a treatment plan for each individual patient. While more patients are being treated with observation for this disease, perhaps the most exciting and game-changing developments will come from genetic studies of these tumors. Once the pathophysiology of this disease is better understood, clinicians can better guide patients 
in treatment recommendations and risk stratification.

\section{ACKNOWLEDGMENTS}

No funding or sponsorship was received for this study or publication of this article. All authors had full access to all of the data in this study and take complete responsibility for the integrity of the data and accuracy of the data analysis. All named authors meet the International Committee of Medical Journal Editors (ICMJE) criteria for authorship for this manuscript, take responsibility for the integrity of the work as a whole, and have given final approval for the version to be published.

Disclosures. J. H. Howard and R. E. Pollock have nothing to disclose.

Compliance with Ethics Guidelines. This article is based on previously conducted studies and does not involve any new studies of human or animal subjects performed by any of the authors.

Open Access. This article is distributed under the terms of the Creative Commons Attribution-NonCommercial 4.0 International License (http://creativecommons.org/licenses/ by-nc/4.0/), which permits any noncommercial use, distribution, and reproduction in any medium, provided you give appropriate credit to the original author(s) and the source, provide a link to the Creative Commons license, and indicate if changes were made.

\section{REFERENCES}

1. Sakorafas GH, Nissotakis C, Peros G. Abdominal desmoid tumors. Surg Oncol. 2007;16(2):131-42.
2. Clark SK, Phillips RK. Desmoids in familial adenomatous polyposis. $\mathrm{Br} \mathrm{J}$ Surg. 1996;83(11):1494-504.

3. de Bree E, Keus R, Melissas J, Tsiftsis D, van Coevorden F. Desmoid tumors: need for an individualized approach. Expert Rev Anticancer Ther. 2009;9(4):525-35.

4. Eastley NC, Hennig IM, Esler CP, Ashford RU. Nationwide trends in the current management of desmoid (aggressive) fibromatosis. Clin Oncol (R Coll Radiol). 2015;27(6):362-8.

5. Kasper B, Baumgarten C, Bonvalot S, et al. Management of sporadic desmoid-type fibromatosis: a European consensus approach based on patients' and professionals' expertise-a sarcoma patients EuroNet and European Organisation for Research and Treatment of Cancer/Soft Tissue and Bone Sarcoma Group initiative. Eur J Cancer. 2015;51(2):127-36.

6. Eastley N, Aujla R, Silk R, et al. Extra-abdominal desmoid fibromatosis-a sarcoma unit review of practice, long term recurrence rates and survival. Eur J Surg Oncol. 2014;40(9):1125-30.

7. Walczak BE, Rose PS. Desmoid: the role of local therapy in an era of systemic options. Curr Treat Options Oncol. 2013;14(3):465-73.

8. Church J, Lynch C, Neary P, LaGuardia L, Elayi E. A desmoid tumor-staging system separates patients with intra-abdominal, familial adenomatous polyposis-associated desmoid disease by behavior and prognosis. Dis Colon Rectum. 2008;51(6):897-901.

9. Bertani E, Testori A, Chiappa A, et al. Recurrence and prognostic factors in patients with aggressive fibromatosis. The role of radical surgery and its limitations. World J Surg Oncol. 2012;10:184.

10. Gronchi A, Colombo C, Le Pechoux C, et al. Sporadic desmoid-type fibromatosis: a stepwise approach to a non-metastasising neoplasm-a position paper from the Italian and the French Sarcoma Group. Ann Oncol. 2014;25(3):578-83.

11. Deyrup AT, Tretiakova M, Montag AG. Estrogen receptor-beta expression in extra abdominal fibromatoses: an analysis of 40 cases. Cancer. 2006;106(1):208-13.

12. Fiore M, Coppola S, Cannell AJ, et al. Desmoid-type fibromatosis and pregnancy: a multi-institutional analysis of recurrence and obstetric risk. Ann Surgery. 2014;259(5):973-8.

13. Peng PD, Hyder O, Mavros MN, et al. Management and recurrence patterns of desmoids tumors: a 
multi-institutional analysis of 211 patients. Ann Surg Oncol. 2012;19(13):4036-42.

14. Crago AM, Denton B, Salas S, et al. A prognostic nomogram for prediction of recurrence in desmoid fibromatosis. Ann Surg. 2013;258(2):347-53.

15. Salas S, Dufresne A, Bui B, et al. Prognostic factors influencing progression-free survival determined from a series of sporadic desmoid tumors: a wait-and-see policy according to tumor presentation. J Clin Oncol. 2011;29(26):3553-8.

16. Gronchi A, Casali PG, Mariani L, et al. Quality of surgery and outcome in extra-abdominal aggressive fibromatosis: a series of patients surgically treated at a single institution. J Clin Oncol. 2003;21(7):1390-7.

17. Huang K, Wang CM, Chen JG, et al. Prognostic factors influencing event-free survival and treatments in desmoid-type fibromatosis: analysis from a large institution. Am J Surg. 2014;207(6):847-54.

18. Lev D, Kotilingam D, Wei C, et al. Optimizing treatment of desmoid tumors. J Clin Oncol. 2007;25(13):1785-91.

19. Salas S, Brulard C, Terrier P, et al. Gene expression profiling of desmoid tumors by cDNA microarrays and correlation with progression-free survival. Clin Cancer Res. 2015;21(18):4194-200.

20. Jilong Y, Jian W, Xiaoyan Z, Xiaoqiu L, Xiongzeng $Z$. Analysis of APC/beta-catenin genes mutations and Wnt signalling pathway in desmoid-type fibromatosis. Pathology. 2007;39(3):319-25.

21. Lips DJ, Barker N, Clevers H, Hennipman A. The role of APC and beta-catenin in the aetiology of aggressive fibromatosis (desmoid tumors). Eur J Surg Oncol. 2009;35(1):3-10.

22. Colombo C, Miceli R, Lazar AJ, et al. CTNNB1 45F mutation is a molecular prognosticator of increased postoperative primary desmoid tumor recurrence: an independent, multicenter validation study. Cancer. 2013;119(20):3696-702.

23. Crago AM, Chmielecki J, Rosenberg M, et al. Near universal detection of alterations in CTNNB1 and Wnt pathway regulators in desmoid-type fibromatosis by whole-exome sequencing and genomic analysis. Genes Chromosomes Cancer. 2015;54(10):606-15.

24. Domont J, Salas S, Lacroix L, et al. High frequency of beta-catenin heterozygous mutations in extra-abdominal fibromatosis: a potential molecular tool for disease management. $\mathrm{Br} \mathrm{J}$ Cancer. 2010;102(6):1032-6.
25. Huss S, Nehles J, Binot E, et al. $\beta$-catenin (CTNNB1) mutations and clinicopathological features of mesenteric desmoid-type fibromatosis. Histopathology. 2013;62(2):294-304.

26. Lazar AJ, Tuvin D, Hajibashi S, et al. Specific mutations in the beta-catenin gene (CTNNB1) correlate with local recurrence in sporadic desmoid tumors. Am J Pathol. 2008;173(5):1518-27.

27. Mullen JT, DeLaney TF, Rosenberg AE, et al. $\beta$-Catenin mutation status and outcomes in sporadic desmoid tumors. Oncologist. 2013;18(9):1043-9.

28. van Broekhoven DL, Verhoef C, Grunhagen DJ, et al. Prognostic value of CTNNB1 gene mutation in primary sporadic aggressive fibromatosis. Ann Surg Oncol. 2015;22(5):1464-70.

29. Desurmont T, Lefevre JH, Shields C, Colas C, Tiret E, Parc Y. Desmoid tumour in familial adenomatous polyposis patients: responses to treatments. Fam Cancer. 2015;14(1):31-9.

30. Huang K, Fu H, Shi YQ, Zhou Y, Du CY. Prognostic factors for extra-abdominal and abdominal wall desmoids: a 20-year experience at a single institution. J Surg Oncol. 2009;100(7):563-9.

31. Mullen JT, Delaney TF, Kobayashi WK, et al. Desmoid tumor: analysis of prognostic factors and outcomes in a surgical series. Ann Surg Oncol. 2012;19(13):4028-35.

32. Pencavel T, Strauss DC, Thomas JM, Hayes AJ. The surgical management of soft tissue tumours arising in the abdominal wall. Eur J Surg Oncol. 2010;36(5):489-95.

33. Wilkinson MJ, Chan KE, Hayes AJ, Strauss DC. Surgical outcomes following resection for sporadic abdominal wall fibromatosis. Ann Surg Oncol. 2014;21(7):2144-9.

34. Wilkinson MJ, Fitzgerald JE, Thomas JM, Hayes AJ, Strauss DC. Surgical resection for non-familial adenomatous polyposis-related intra-abdominal fibromatosis. Br J Surg. 2012;99(5):706-13.

35. Latchford AR, Sturt NJ, Neale K, Rogers PA, Phillips RK. A 10-year review of surgery for desmoid disease associated with familial adenomatous polyposis. $\mathrm{Br}$ J Surg. 2006;93(10):1258-64.

36. Chatzipetrou MA, Tzakis AG, Pinna AD, et al. Intestinal transplantation for the treatment of desmoid tumors associated with familial adenomatous polyposis. Surgery. 2001;129(3):277-81.

37. Jovine E, Masetti M, Cautero N, et al. Modified multivisceral transplantation without a liver graft 
for Gardner/desmoid syndrome and chronic intestinal pseudo-obstruction. Transplant Proc. 2002;34(3):911-2.

38. Nikeghbalian S, Aliakbarian $M$, Shamsaeefar A, Kazemi K, Bahreini A, Malekhosseini SA. Multivisceral transplantation for the treatment of intra-abdominal tumors. Transplant Proc. 2013;45(10):3528-30.

39. Bonvalot $S$, Eldweny $H$, Haddad $V$, et al. Extra-abdominal primary fibromatosis: aggressive management could be avoided in a subgroup of patients. Eur J Surg Oncol. 2008;34(4):462-8.

40. Fiore M, Rimareix F, Mariani L, et al. Desmoid-type fibromatosis: a front-line conservative approach to select patients for surgical treatment. Ann Surg Oncol. 2009;16(9):2587-93.

41. Nuyttens JJ, Rust PF, Thomas CR Jr, Turrisi AT 3rd. Surgery versus radiation therapy for patients with aggressive fibromatosis or desmoid tumors: a comparative review of 22 articles. Cancer. 2000;88(7):1517-23.

42. Ballo MT, Zagars GK, Pollack A. Radiation therapy in the management of desmoid tumors. Int J Radiat Oncol Biol Phys. 1998;42(5):1007-14.

43. Guadagnolo BA, Zagars GK, Ballo MT. Long-term outcomes for desmoid tumors treated with radiation therapy. Int J Radiat Oncol Biol Phys. 2008;71(2):441-7.

44. Keus RB, Nout RA, Blay JY, et al. Results of a phase II pilot study of moderate dose radiotherapy for inoperable desmoid-type fibromatosis-an EORTC STBSG and ROG study (EORTC 62991-22998). Ann Oncol. 2013;24(10):2672-6.

45. Micke O, Seegenschmiedt $\mathrm{MH}$, German Cooperative Group on Radiotherapy for Benign Diseases. Radiation therapy for aggressive fibromatosis (desmoid tumors): results of a national patterns of care study. Int J Radiat Oncol Biol Phys. 2005;61(3):882-91.

46. Gluck I, Griffith KA, Biermann JS, Feng FY, Lucas DR, Ben-Josef E. Role of radiotherapy in the management of desmoid tumors. Int $\mathrm{J}$ Radiat Oncol Biol Phys. 2011;80(3):787-92.

47. van Broekhoven DL, Verhoef C, Elias SG, et al. Local recurrence after surgery for primary extra-abdominal desmoid-type fibromatosis. $\mathrm{Br} \mathrm{J}$ Surg. 2013;100(9):1214-9.

48. de Camargo VP, Keohan ML, D'Adamo DR, et al. Clinical outcomes of systemic therapy for patients with deep fibromatosis (desmoid tumor). Cancer. 2010;116(9):2258-65.
49. Hansmann A, Adolph C, Vogel T, Unger A, Moeslein G. High-dose tamoxifen and sulindac as first-line treatment for desmoid tumors. Cancer. 2004;100(3):612-20.

50. Tanaka K, Yoshikawa R, Yanagi H, et al. Regression of sporadic intra-abdominal desmoid tumour following administration of non-steroidal anti-inflammatory drug. World J Surg Oncol. 2008;6:17.

51. Bocale D, Rotelli MT, Cavallini A, Altomare DF. Anti-oestrogen therapy in the treatment of desmoid tumours: a systematic review. Colorectal Dis. 2011;13(12):e388-95.

52. Quast DR, Schneider R, Burdzik E, Hoppe S, Möslein G. Long-term outcome of sporadic and FAP-associated desmoid tumors treated with high-dose selective estrogen receptor modulators and sulindac: a single-center long-term observational study in 134 patients. Fam Cancer. 2016;15(1):31-40.

53. Gega M, Yanagi H, Yoshikawa R, et al. Successful chemotherapeutic modality of doxorubicin plus dacarbazine for the treatment of desmoid tumors in association with familial adenomatous polyposis. J Clin Oncol. 2006;24(1):102-5.

54. Okuno SH, Edmonson JH. Combination chemotherapy for desmoid tumors. Cancer. 2003;97(4):1134-5.

55. Patel SR, Evans HL, Benjamin RS. Combination chemotherapy in adult desmoid tumors. Cancer. 1993;72(11):3244-7.

56. Garbay D, Le Cesne A, Penel N, et al. Chemotherapy in patients with desmoid tumors: a study from the French Sarcoma Group (FSG). Ann Oncol. 2012;23(1):182-6.

57. Azzarelli A, Gronchi A, Bertulli R, et al. Low-dose chemotherapy with methotrexate and vinblastine for patients with advanced aggressive fibromatosis. Cancer. 2001;92(5):1259-64.

58. Bertagnolli MM, Morgan JA, Fletcher CD, et al. Multimodality treatment of mesenteric desmoid tumours. Eur J Cancer. 2008;44(16):2404-10.

59. Weiss AJ, Horowitz S, Lackman RD. Therapy of desmoid tumors and fibromatosis using vinorelbine. Am J Clin Oncol. 1999;22(2):193-5.

60. Chugh R, Wathen JK, Patel SR, et al. Efficacy of imatinib in aggressive fibromatosis: results of a phase II multicenter Sarcoma Alliance for Research through Collaboration (SARC) trial. Clin Cancer Res. 2010;16(19):4884-91. 
61. Heinrich MC, McArthur GA, Demetri GD, et al. Clinical and molecular studies of the effect of imatinib on advanced aggressive fibromatosis (desmoid tumor). J Clin Oncol. 2006;24(7):1195-203.

62. Penel N, Le Cesne A, Bui BN, et al. Imatinib for progressive and recurrent aggressive fibromatosis (desmoid tumors): an FNCLCC/French Sarcoma Group phase II trial with a long-term follow-up. Ann Oncol. 2011;22(2):452-7.

63. Jo JC, Hong YS, Kim KP, et al. A prospective multicenter phase II study of sunitinib in patients with advanced aggressive fibromatosis. Invest New Drugs. 2014;32(2):369-76.

64. Gounder MM, Lefkowitz RA, Keohan ML, et al. Activity of Sorafenib against desmoid tumor/deep fibromatosis. Clin Cancer Res. 2011;17(12):4082-90.

65. Martin-Liberal J, Benson C, McCarty H, Thway K, Messiou C, Judson I. Pazopanib is an active treatment in desmoid tumour/aggressive fibromatosis. Clin Sarcoma Res. 2013;3(1):13.

66. Shang H, Braggio D, Lee YJ, et al. Targeting the Notch pathway: a potential therapeutic approach for desmoid tumors. Cancer. 2015;121(22):4088-96.

67. Messersmith WA, Shapiro GI, Cleary JM, et al. A Phase I, dose-finding study in patients with advanced solid malignancies of the oral gamma-secretase inhibitor PF-03084014. Clin Cancer Res. 2015;21(1):60-7.

68. Hughes DP, Kummar S, Lazar AJ. New, tolerable gamma-secretase inhibitor takes desmoid down a notch. Clin Cancer Res. 2015;21(1):7-9.

69. Briggs A, Rosenberg L, Buie JD, Rizvi H, Bertagnolli MM, Cho NL. Antitumor effects of hyaluronan inhibition in desmoid tumors. Carcinogenesis. 2015;36(2):272-9. 